\title{
EL DISTRÉS PSICOLÓGICO Y ABUSO DE DROGAS EN PACIENTES EN CENTROS DE TRATAMIENTO DEL GRAN VALPARAÍSO - CHILE: IMPLICACIONES PARA LAS POLÍTICAS Y LOS PROGRAMAS
}

\author{
Liliana Basso Musso', Robert Mann², Carol Strike ${ }^{3}$, Bruna Brands ${ }^{4}$, Akwatu Khenti $^{5}$
}

\begin{abstract}
${ }^{1}$ MSc. Profesor Titular. Universidad de Valparaíso. Chile. E-mail: lbassom@hotmail.com
${ }^{2}$ Ph.D. Investigator. Centre for Addiction and Mental Health. University of Toronto. Canada. E-mail: robert_mann@camh.net

${ }^{3}$ Ph.D. Investigator. Health Systems Research and Consulting Unit. Centre for Addiction and Mental Health. University of Toronto. Canada. E-mail: carol_strike@camh.net

${ }^{4}$ Ph.D. Investigator. Office of Research and Surveillance. Drug Strategy and Controlled Substances Programme. Health Canada and Public Health and Regulatory Policies. Center for Addiction and Mental Health. University of Toronto. Canada. E-mail: bruna_brands@camh.net

${ }^{5}$ MSc. Director International Health Office. Centre for Addiction and Mental Health. Toronto, Canada. E-mail: akwatu_khenti@ camh.net
\end{abstract}

RESUMEN: El objetivo fue determinar la prevalencia de comorbilidad entre distrés psicológico y abuso/dependencia de drogas en pacientes en centros de tratamiento. Este estudio epidemiológico, multicéntrico, de corte transversal, realizado en 107 pacientes mayor es de 18 años, en tratamiento por abuso/dependencia de drogas, en centros de tratamiento de Valparaíso, Chile, mostró además otros factores involucrados como: aspectos sociodemográficos, funcionalidad familiar, características del consumo de drogas y aspectos de la atención otorgada. Para recolección de datos se usó el EULAC (Instrumento para diagnostico rápido de situación de tratamiento para problemas por consumo de drogas en el ámbito local) el K10 y Apgar familiar. Resultados: 48.6\% presentaron distrés psicológico; 88.8\% refirieron satisfacción por el tratamiento actual. $66.4 \%$ pertenece a una familia normo funcional. Los resultados obtenidos permitirán mejorar las intervenciones en pacientes que están en tratamiento y rehabilitación en los centros de tratamiento.

DESCRIPTORES: Comorbilidad. Trastornos relacionados con sustancias. Distrés psicológico. Atención en salud mental.

\section{THE PSYCHOLOGICAL DISTRESS AND DRUG ABUSE AMONG PATIENTS IN TREATMENT CENTRES, IN AREA OF VALPARAISO - CHILE: IMPLICATIONS FOR POLICY AND PROGRAMS}

\begin{abstract}
The objective was to determine the prevalence of comorbidity among psychological distress and abuse/dependence on drugs on patients in treatment centres. This epidemiological, multicentre, study of cross-section, 107 patients over 18 years old, in treatment by abuse/dependence on drugs, Valparaiso, Chile, treatment centres, showed also other factors involved such as: aspects sociodemographic, functionality family, drug features and aspects of the granted services. The EULAC (Instrument for rapid diagnosis of situation of treatment for problems drug locally) the $10 \mathrm{~K}$ and Family Apgar was used for data collection. Results: $48.6 \%$ presented psychological distréss; $88.8 \%$ spoke satisfaction by current treatment; $66.4 \%$ belongs to a family normal functional. The results will enable to improve interventions in patients who are in treatment and rehabilitation in treatment centres.
\end{abstract}

DESCRIPTORS: Comorbidity. Substance use-related disorders. Psychological distress. Mental health assistance.

\section{O SOFRIMENTO PSÍQUICO E ABUSO DE DROGAS EM PACIENTES DE CENTROS DE TRATAMENTO NA ÁREA DE VALPARAÍSO - CHILE: IMPLICAÇÕES PARA AS POLÍTICAS E PROGRAMAS}

RESUMO: O objetivo foi determinar a prevalência de comorbilidade entre sofrimento psiquico e abuso/dependência de drogas em pacientes em centros de tratamento de Valparaiso, Chile. Estudo epidemiológico, multicêntrico, de corte transversal realizado com 107 pacientes com idade acima de 18 anos, internados nos centros de tratamento de Valparaíso, Chile. Analisou-se os seguintes aspectos: aspectos sociodemográficos, funcionalidade da família, caracteristica do consumo de drogas e qualidade dos serviços oferecidos para tratamento. A EULAC (Instrumento de diagnóstico rápido de situação de tratamento para drogas problemas localmente) a $10 \mathrm{~K}$ e o Apgar familiar foi usado para coleta de dados. Resultaram que 48,6\% apresentados stress; $88,8 \%$ relataram satisfação pelo tratamento atual; $66,4 \%$ pertence a uma família Normofuncional. Os resultados permitirão melhorar as intervenções em pacientes que estão em tratamento e reabilitação em centros de tratamento.

DESCRITORES: Comorbidade. Transtornos relacionados ao uso de substancias. Sofrimento psíquico. Assistência em saúde mental 


\section{INTRODUCCIÓN}

La co-ocurrencia de trastornos entre abuso y dependencia de sustancias psicoactivas y otros problemas de salud mental, es frecuente. Existe alto riesgo de consumo de drogas en pacientes con trastornos mentales y una alta frecuencia de psicopatología, desencadenada por abuso de drogas ilícitas y alcohol. La presencia simultánea de ambos trastornos se conoce como comorbilidad, y afecta la calidad de vida de los pacientes. ${ }^{1}$ La alta tasa de comorbilidad entre trastornos por uso de sustancias y otros trastornos mentales, como ansiedad, depresión o distrés psicológico, puede relacionarse con la compleja interacción entre medio ambiente, características de las drogas y factores individuales. ${ }^{2}$ La importancia de ampliar el campo de la investigación en relación con comorbilidad, asociada al abuso de alcohol y drogas ilícitas y distrés psicológico, adquiere especial interés cuando se relaciona entonces, con el individuo, la familia, al lugar de trabajo, la escuela, la elaboración de políticas sociales y económicas, las implicaciones culturales, las características propias de las drogas y el medio ambiente, elementos que interactúan en un paciente con comorbilidad. ${ }^{3}$

Dentro de las características familiares, la estructura y el funcionamiento familiar pueden promover o desfavorecer la presencia de comorbilidad, contribuyendo a generar un contexto protector o de riesgo. ${ }^{4}$ Las propiedades especificas de las drogas (tipo de droga consumida, edad de inicio, vía de consumo), contribuyen a la aparición de un patrón de consumo, abuso y/o dependencia. ${ }^{5}$ Por otro lado, las características socio demográficas de la persona, edad, género, nivel educativo, empleo, raza o étnia, estado marital, ingresos económicos y la presencia o no de distrés psicológico y sus niveles, aportan otro componente de la comorbilidad. ${ }^{6}$ También confluyen factores judiciales: antecedentes de problemas judiciales relacionados con el consumo de drogas; y sanitarios: los Centros de Tratamiento. Por tanto, cuando el abuso de sustancias y otro desorden mental y/o distrés psicológico ocurre simultáneamente, existe mayor incapacidad funcional y conductas autodestructivas, por lo que el éxito del tratamiento se vuelve más problemático. El tipo de atención con que cuentan, el equipo de profesionales que los atiende, la satisfacción del usuario con esta atención, son aspectos fundamentales que permiten determinar el funcionamiento de los Centros de Tratamiento y si ofrecen cuidados para la comorbilidad.
De acuerdo al General Surgeon Report sobre salud mental de los Estados Unidos, entre el 31 a $65 \%$ de los individuos que habían abusado de sustancias alguna vez en la vida, también tenían una historia de al menos un desorden mental, y $51 \%$ de los mismos, con uno o más desordenes de salud mental a lo largo de su vida, tenía al menos una historia de trastornos por abuso de sustancia. ${ }^{7}$ Datos del Área de Captación de Estudios Epidemiológico, revelaron que alrededor de la mitad de los individuos diagnosticados con abuso o dependencia de alcohol u otras drogas, también tienen un diagnóstico psiquiátrico. La presencia de depresión mayor entre los que usan drogas varía entre $30 \%$ a $50 \%$. Datos similares también fueron hallados en publicaciones más recientes en todo el mundo. ${ }^{8-9}$ Se afirma que la prevalencia de comorbilidad entre la depresión y el consumo de alcohol es de $15 \%$ a $67 \% .^{10} \mathrm{El}$ interés en la coocurrencia de trastornos mentales y consumo de sustancias es importante porque que afecta el curso, el tratamiento y el pronóstico de ambos trastornos negativamente. ${ }^{11-12}$ La presencia de ambos problemas de forma simultánea, produce déficit cognitivo, problemas interpersonales, daño emocional y biológico. ${ }^{13}$

El impacto de estos dos problemas en la misma persona, también tiene un efecto sobre sus familias; y suele producir sentimientos de vergüenza, culpa, estigma, y desesperación. ${ }^{14}$ Estos sentimientos también, deben abordarse en el tratamiento, como lo demuestran estudios, con intervenciones integradas y tratamiento para ambos problemas..$^{15}$ En la relación entre funcionamiento familiar y consumo de sustancias en adolescentes psiquiátricos, se encontró que el mayor grado de insatisfacción de la familia en los ámbitos de respuesta afectiva y función del rol, se asoció con niveles más altos de abuso de sustancias. ${ }^{16}$ Algunos estudios sugieren que la comorbilidad debe ser investigada, ya que se encontró que su tasa es elevada en los Centros de Tratamiento para el abuso de drogas, porque las personas que tienen ambos problemas, buscan el tratamiento más frecuentemente que aquellos que sólo tienen un problema. ${ }^{17}$ Casi todos los estudios sobre comorbilidad se llevaron a cabo en América del Norte, Europa y Australia. Si bien, se sabe que la prevalencia está influenciada por género, etnia y otras variables, ${ }^{1-10}$ hay pocos estudios desarrollados en América Latina y el Caribe.

Chile no está ajeno a esta realidad, pese a su desarrollo en las políticas y programas para 
afrontar el fenómeno de las drogas. En 2001 se implementó el Programa para Tratamiento y Rehabilitación de Personas con Dependencia de alcohol y drogas, que abarca los servicios de salud, incluida la red de atención primaria, los centros comunitarios de Salud Mental, hospitales y comunidades terapéuticas en todo el territorio nacional. Este programa ofrece gratuitamente cobertura y tratamiento a beneficiarios del Fondo Nacional de Salud (FONASA), en cuatro modalidades que varían en función de las características, necesidades y complejidad de cada caso. El programa es un esfuerzo conjunto del Ministerio de Salud y el Consejo Nacional para el Control de Estupefacientes (CONACE), que depende del Ministerio del Interior y del Ministerio de Justicia. ${ }^{18}$

La misión del CONACE es implementar políticas públicas sobre la problemática de drogas, y prevenir el consumo y tráfico de sustancias ilícitas en el país. Los principales estudios en relación a la prevalencia del uso de drogas en Chile, son efectuados por CONACE, que los realiza sobre la base de muestras probabilísticas. Esto ha permitido comparaciones en la prevalencia de vida, en el consumo de drogas de los últimos 12 meses y consumo en últimos 30 días. El consumo dado en los últimos 12 meses por el último estudio 2008, arroja lo siguiente: alcohol: $68.5 \%$, mariguana: $6.4 \%$, cocaína pasta base: $0.7 \%$, cocaína polvo: $1.8 \%$, crack: $0.2 \%$, éxtasis: $0.1 \% \cdot{ }^{19}$ Hay pocos datos sobre la prevalencia de los trastornos mentales, Una pesquisa. mostrar lo siguiente: (prevalencia de vida) principales trastornos de salud mental: $36.0 \%$. agorafobia: $11.1 \%$, depresión mayor: $9.0 \%$ distímia: $8.0 \%$, dependencia de alcohol: $6.4 \% .{ }^{20} \mathrm{En}$ Chile no existen estudios de comorbilidad entre trastornos de salud mental y abuso o dependencia a drogas, por lo que este estudio contribuirá a tener una aproximación actualizada del problema.

El objetivo de esta investigación fue determinar la prevalencia de comorbilidad entre distrés psicológico y abuso/dependencia de drogas en pacientes en Centros de Tratamiento. Los enfermeros están participando activamente en equipos multidisciplinarios que atienden a estos pacientes en los Centros de Tratamiento, y en el área de atención general de salud, razón por la que deben estar atentos en la gestión de los cuidados de enfermería, a conocer y considerar aspectos derivados de la comorbilidad, que acentúan la problemática del consumo de drogas y sus efectos, complejizando sus tratamientos.

\section{METODOLOGÍA}

Este estudio es parte de un estudio epidemiológico, multicéntrico, de corte transversal, dirigido a población mayor de 18 años, que está en tratamiento por abuso/dependencia de drogas, efectuado en Panamá, Guatemala, Brasil, Uruguay, Paraguay, Jamaica y Chile. El estudio en el Gran Valparaíso, Chile, fue realizado en 10 Centros de Tratamiento y Rehabilitación para usuarios/pacientes con abuso o dependencia del consumo de drogas, contuición técnica del CONACE.

La muestra fue de 107 usuarios, los que se seleccionaron en base a su voluntad de participar, siendo ésta la única condición. Se consideró a los que estaban presentes en cada Centro en el momento en que concurrió el investigador a pasar el instrumento y que habían sido consultados previamente por sus encargados. La muestra fue calculada con un poder para la detección de al menos $40 \%$ de prevalencia de distrés psicológico severo y muy severo, considerando un punto de corte de 22 en la escala K-10. ${ }^{28}$ La muestra sería capaz de detectar una diferencia mínima del $5 \%$ en la prevalencia, y el mantenimiento de los criterios de errores de tipo I y II en 0.05 y 0.20 respectivamente. Los datos se obtuvieron través del formulario EULAC-CICAD (Instrumento para diagnostico rápido de situación de tratamiento para problemas por consumo de drogas en el ámbito local) adaptado para este estudio por los investigadores. Consta de 30 preguntas sobre estatus sociodemográfico, historia de abuso de drogas y alcohol, antecedentes judiciales, problemas mentales pasados y actuales, y algunos aspectos sobre los Centros de Tratamiento. El distrés psicológico se evaluó mediante el Instrumento Kessler-10 (K-10), ${ }^{6}$ cuestionario de 10 preguntas que mide distrés psicológico.

La clasificación para distrés psicológico se hizo en cuatro grupos: bajo (10-15), moderado (16$21)$, severo (22-29) y muy severo (30-50). A efectos analíticos, la mayoría de los estudios unen las dos últimas categorías (distrés severo y muy severo). Para la estimación de la percepción de funcionalidad familiar, se aplicó el Instrumento APGAR familiar. ${ }^{21}$ Los valores de las escalas van de cero a 10 y se basa en la percepción de satisfacción de los participantes de las relaciones dentro de su familia. Incluye cinco aspectos de la función familiar: adaptabilidad, asociación, crecimiento, afecto y resolución de conflictos. La escala ha sido valida$\mathrm{da}^{22} \mathrm{y}$ es ampliamente utilizada. La interpretación del APGAR familiar se basa en la puntuación, de cada pregunta cuyos valores van de cero a dos. Una puntuación total de siete o más, sugiere una 
percepción de alta funcionalidad familiar, entre cuatro y seis, sugiere una moderada disfunción familiar, y tres o menos, una severa disfunción familiar. Se utilizó Epi Info versión 3.5.1 (Center for Disease Control and Prevention: CDC, 2008) para introducir y analizar los datos.

El estudio fue aprobado por la Oficina de Ética en Investigación del Centro de Adicciones y Salud Mental de Canadá (CAMH) Protocol Reference \# 225/2008, y por el Comité de Ética de Investigación de la Universidad de Valparaíso, Chile. Los Usuarios que estuvieron de acuerdo en participar, lo hicieron voluntariamente y firmaron el consentimiento informado respectivo. La recolección de información en los Centros de Tratamiento desarrollada entre noviembre 2009 y marzo 2010, fue efectuada por el investigador principal. Los cuestionarios usados y consentimientos informados firmados por los usuarios, fueron resguardados en dos cajas separadas, para garantizar su seguridad y confidencialidad.

\section{RESULTADOS}

\section{Aspectos socio-demográficos}

De los usuarios, $64.6 \%(n=69)$ fueron hombres, siendo menor el porcentaje de mujeres con $35.8 \%$ ( $n=39)$; sus edades en mayor medida según el rango mostrado, estuvo entre 24 y 30 años 62.9 $\%(\mathrm{n}=66)$. En cuanto al lugar de residencia, y esto tiene relación con el lugar de ubicación de los Centros de Tratamiento donde $62.9 \%(n=73)$ de la muestra vive en Viña del Mar. La gran mayoría $(99.1 \%)(n=106)$ son de nacionalidad chilena. Del grupo étnico, $56.6 \%(\mathrm{n}=60)$ de los consultados se considera blanco y $40.6 \%(n=43)$ se considera mixto. Han vivido los últimos 30 días en casa de su familia de origen $(36.9 \%)(n=41)$, seguido de $23.4 \%$ $(n=26)$ que vive en su propia casa, de la que es propietario; $22.5 \%(\mathrm{n}=25)$ que también vive en su propia casa, pero es arrendatario. De la población, $27 \%(n=54)$ vive con otro familiar, $22.5 \%(n=45)$ vive con su madre, $13.0 \%(n=26)$ con un hermano y $10.5 \%(n=21)$ con el novio/a.

\section{Características educacionales y ocupacionales}

De los usuarios, $98.1 \%(n=105)$ sabe leer y escribir, y este resultado es congruente con el nivel educacional en Chile, pudiendo observarse además, que $32.1 \%(n=34)$ ha terminado su Educación Secundaria completa, $17.9 \%(\mathrm{n}=19)$ tiene Educación Secundaria incompleta, $16.0 \%(n=17)$ ha tenido educación universitaria completa, y con el mismo número y porcentaje, es decir, $16 \%(n=17)$, educación universitaria incompleta. Por otro lado, solo $1.9 \%(n=2)$ no ha concurrido nunca a Educación escolar, siendo este porcentaje igual al que aparece de los Usuarios que no saben leer ni escribir. En relación a la Actividad laboral, 51.4\% $(n=56)$ tiene un trabajo ya sea empleado o autónomo, $14.7 \%$ $(n=16)$ son dueñas de casa y el $29.4 \%(n=32)$ está desempleado, lo que es superior a la tasa de desempleo en el Gran Valparaíso, Chile, que alcanza a alrededor de un $10 \%$. De los ingresos percibidos: $42.1 \%(n=40)$ no percibe ingresos; $9.5 \%(n=9)$ recibe menos de US $\$ 200.00,23.2 \%(n=22)$ recibe entre US\$ 200.00 y US\$ 400.00, y $25.3 \%(n=24)$ declara recibir ingresos mayores a US\$ 400.00 .

\section{Problemas legales relacionados con alcohol o drogas}

De los usuarios, $48.5 \%(n=50)$ ha tenido problemas legales relacionado con alcohol o drogas y $51.5 \%(n=53)$ no los ha tenido. En el último año $32.4 \%(n=33)$ ha tenido problemas legales y $67.6 \%$ $(n=69)$ no los ha tenido. En relación a las veces que ha tenido problemas legales en el último año: $69.7 \%(n=69)$ no ha tenido, $14.1 \%(n=14)$ ha tenido una vez, $6.1 \%(n=6)$ dos veces, cuatro, $4.0 \%(n=4)$ los ha tenido tres veces, $3 \%(n=3)$ cuatro veces; cinco, ocho y 20 veces solo los ha tenido $1 \%(n=1)$.

\section{Referencia a los centros de tratamiento}

La mayoría de los usuarios refiere concurrir a los Centros de Tratamiento, voluntariamente $(32.3 \%)(n=50)$; otras formas de referencias son: Por sugerencia de familiares y amigos, con $20 \%$ $(n=31)$; de otro programa de tratamiento, con $19.4 \%(n=30)$; de unidad primaria de salud, con $10.3 \%(n=16)$; del Consejo nacional de drogas, con $6.5 \%(n=10)$; desde una Unidad secundaria o terciaria 5.8\% (n=9); y de la Justicia o Policía, con $3.2 \%(n=5)$. Los más bajos porcentajes de referencia pertenecen a servicios sociales con $1.3 \%(n=2)$; por el patrón, y también por el sistema escolar con $0.6 \%(n=1)$. Las Prisiones y Centros de Detención juvenil en Chile, no hacen referencias de usuarios a los Centros de Atención usados en este estudio.

\section{Tratamientos previos de los pacientes antes de su atención en los centros de tratamiento}

En cuanto a si ha recibido tratamiento por abuso o dependencia a drogas antes del ingreso al Centro, 35.9\% ( $\mathrm{n}=37)$ no ha tenido tratamientos 
anteriores; $33 \%(n=34)$ solo ha tenido una atención previa; y $31.1 \%(n=32)$ ha tenido más de una atención. El tipo de atención por abuso de drogas, más recibido fue: atención en consulta externa, con 30.3\% $(n=43)$; seguido por Atención dada en servicio residencial, con $10.6 \%(n=15)$; en unidad de desintoxicación, con 9.2\% ( $n=13)$; en unidad de internación especializada en tratamiento de drogas, con $9.0 \%$ $(\mathrm{n}=14)$; en unidad psiquiátrica, con $7.7 \%(\mathrm{n}=11)$; con atención diaria, con $4.2 \%(\mathrm{n}=6)$; $y$ asistiendo a grupos de autoayuda, cinco, con 3.5\% ( $\mathrm{n}=5)$.

La Tabla 1 describe que las drogas más usadas por los usuarios, en los últimos 30 días antes de ingresar a los Centros de tratamiento, fue: alcohol, con $26.6 \%(\mathrm{n}=79)$, cocaína polvo, con $18.2 \%(n=54)$, mariguana, con $17.2 \%(n=51)$ y pasta de coca, con $16.9 \%(n=50)$, mostrando resultados que reflejan el consumo de las drogas que causan mayores problemas de Salud Pública en Chile. Aparece además 5.1\% $(\mathrm{n}=15)$ usuarios haciendo uso de benzodiacepinas sin indicación médica. El resto de los resultados no tiene relevancia en comparación con los anteriormente mencionados.

Tabla 1 - Distribución de las drogas consumidas durante los últimos 30 días antes de iniciar el tratamiento de los pacientes atendidos en Centros de tratamiento del Gran ValparaísoChile, 2010

\begin{tabular}{lrr}
\hline $\begin{array}{l}\text { Drogas consumidas durante } \\
\text { los últimos } 30 \text { días antes de } \\
\text { iniciar el tratamiento }\end{array}$ & Frecuencia & $\%$ \\
\hline Abuso de medicación prescrita & 7 & 2.4 \\
Alcohol & 79 & 26.6 \\
Anfetaminas (uso no prescrito) & 13 & 4.4 \\
Benzodiacepinas & 15 & 5.1 \\
Cannabis (marihuana, ganja y & 51 & 17.2 \\
otros derivados) & 54 & 18.2 \\
Cocaína & 2 & 0.7 \\
Crack & 3 & 1.0 \\
Esteroides anabólicos & 2 & 0.7 \\
Heroína & 4 & 1.4 \\
Inhalables & 3 & 1.0 \\
LSD & - & - \\
Metadona & & \\
Metanfetaminas (éxtasis) y otros & 6 & 2.0 \\
derivados & 2 & 0.7 \\
Opioides & 2 & 1.0 \\
Otros alucinógenos & 3 & 16.9 \\
Pasta de coca (basuco, paco) & 50 & 0.7 \\
Barbitúricos & 296.0 \\
\hline Total & & 100 \\
\hline
\end{tabular}

La tabla 2 indica resultados sobre la primera droga en importancia por la que el usuario concurrió a tratamiento al Centro: pasta de coca o pasta base, con $41 \%(n=43)$; le sigue cocaína polvo, con $25.7 \%(n=27)$; alcohol, con $23.8 \%(n=25) ; y$ a continuación, mariguana, con $5.7 \%(n=6)$. No aparecen los estimulantes, los opioides: heroína y metadona, ni los alucinógenos, inhalantes y abuso de medicación recetada por el médico. Tampoco esteroides anabólicos.

Tabla 2 - Características relacionadas con la primera droga mencionada, utilizada en los últimos 30 días antes del actual tratamiento, por la cual se buscó atención en los Centros de tratamiento del Gran Valparaíso-Chile, 2010

\begin{tabular}{|c|c|c|c|}
\hline Tipo de drogas & Categorías & $\mathbf{n}$ & $\%$ \\
\hline Alcohol & & 25 & 23.8 \\
\hline \multirow[t]{2}{*}{ Mariguana } & & 6 & 5.7 \\
\hline & Polvo & 27 & 25.7 \\
\hline \multirow[t]{3}{*}{ Cocaína } & Pasta de coca & 43 & 41.0 \\
\hline & Crack & - & - \\
\hline & Barbitúricos & 2 & 1.9 \\
\hline \multirow{2}{*}{ Hipnóticos y sedativos } & Benzodiacepinas & - & - \\
\hline & Anfetaminas & - & - \\
\hline \multirow[t]{3}{*}{ Estimulantes } & Metanfetaminas & - & - \\
\hline & Otros & - & - \\
\hline & Heroína & - & - \\
\hline \multirow[t]{2}{*}{ Opioides } & Metadona & - & - \\
\hline & Otros & 2 & 1.9 \\
\hline \multicolumn{2}{|c|}{ Abuso de medicación recetada por el médico } & - & - \\
\hline \multicolumn{2}{|l|}{ Inhalantes } & - & - \\
\hline \multirow{2}{*}{ Alucinógenos } & LSD & - & - \\
\hline & Otros & - & - \\
\hline \multicolumn{2}{|l|}{ Esteroides anabólicos } & - & - \\
\hline \multicolumn{2}{|l|}{ Otras drogas } & - & - \\
\hline \multicolumn{2}{|l|}{ Subtotal } & 105 & 100 \\
\hline \multicolumn{2}{|l|}{ No respondieron } & 2 & \\
\hline \multicolumn{2}{|l|}{ Total } & 107 & \\
\hline
\end{tabular}

En la tabla 3 aparecen los diagnósticos previos y actuales de los pacientes dados en los Centros de Tratamiento: de los diagnósticos previos que han sido dados a los pacientes, está en primer lugar depresión con 45.8\% ( $\mathrm{n}=49)$; luego ansiedad $39.9 \%(n=42)$; y por último, trastorno bipolar con cinco $4.7 \%(n=5)$. En los diagnósticos actuales está en primer lugar la ansiedad con $44.9 \% \quad(n=48)$; luego la depresión con $28 \%(\mathrm{n}=30)$ y por último, el trastorno bipolar con $1.9 \%(n=2)$. En el Centro de 
Tratamiento están recibiendo tratamiento, de los $\quad(n=38)$; diagnosticados con depresión en $76.7 \%$ pacientes diagnosticados con Ansiedad en 79.2\% $\quad(n=23)$; y con trastorno bipolar en $100 \%(n=2)$.

Tabla 3 - Diagnósticos previos, actuales; y tratamientos actuales de los pacientes atendidos en Centros de tratamiento del Gran Valparaíso-Chile, 2010

\begin{tabular}{|c|c|c|c|c|c|c|c|c|c|}
\hline \multirow{2}{*}{ Variables } & \multirow{2}{*}{ Categorías } & \multicolumn{2}{|c|}{ Si } & \multicolumn{2}{|c|}{ No } & \multicolumn{2}{|c|}{ No sabe } & \multicolumn{2}{|c|}{ N R } \\
\hline & & $\mathbf{n}$ & $\%$ & $n$ & $\%$ & $\mathbf{n}$ & $\%$ & $n$ & $\%$ \\
\hline \multirow{5}{*}{ Diagnósticos previos } & Ansiedad & 42 & 39.3 & 44 & 41.1 & 3 & 2.8 & 18 & 16.8 \\
\hline & Depresión & 49 & 45.8 & 41 & 38.3 & 6 & 5.6 & 11 & 10.3 \\
\hline & Esquizofrenia & - & - & 66 & 61.7 & 3 & 2.8 & 38 & 35.5 \\
\hline & Trastorno bipolar & 5 & 4.7 & 62 & 57.9 & 3 & 2.8 & 37 & 34.6 \\
\hline & Otros & 3 & & & & & & & \\
\hline \multirow{5}{*}{ Diagnóstico actual } & Ansiedad & 48 & 44.9 & 41 & 38.3 & - & - & 18 & 16.8 \\
\hline & Depresión & 30 & 28.0 & 50 & 46.7 & - & - & 27 & 25.2 \\
\hline & Esquizofrenia & - & - & 56 & 52.3 & - & - & 51 & 47.6 \\
\hline & Trastorno bipolar & 2 & 1.9 & 55 & 51.4 & - & - & 50 & 46.7 \\
\hline & Otros & 2 & 100 & - & - & - & - & - & - \\
\hline \multirow{5}{*}{ Tratamiento en este centro } & Ansiedad & 38 & 79.2 & 3 & 6.2 & - & - & 7 & 14.6 \\
\hline & Depresión & 23 & 76.7 & 1 & 3.3 & - & - & 6 & 20.0 \\
\hline & Esquizofrenia & - & - & - & - & - & - & - & - \\
\hline & Trastorno bipolar & 2 & 100 & - & - & - & - & - & - \\
\hline & Otros & 2 & 100 & - & - & - & - & - & - \\
\hline
\end{tabular}

La tabla 4 indica la presencia de distrés psicológico mostrado por los pacientes: $20.6 \%(n=22)$ presenta un nivel de distrés bajo; $30.8 \%(n=33)$ nivel de distrés moderado; $24.3 \%(\mathrm{n}=26)$ nivel de distrés severo; $24.3 \%(\mathrm{n}=26)$ de distrés muy severo.
De la funcionalidad familiar percibida, ésta es señalada como normo funcional en $66.4 \%$ $(\mathrm{n}=71)$ pacientes; moderadamente disfuncional en $22.4 \%(n=24)$; y severamente disfuncional en $11.2 \%(\mathrm{n}=12)$.

Tabla 4 - Distrés psicológico y funcionalidad familiar percibida por los pacientes atendidos en Centros de tratamiento del Gran Valparaíso-Chile, 2010

\begin{tabular}{llll}
\hline Variable & Categoría & $\mathbf{S i}$ & $\%$ \\
\hline & Bajo (10-15) & 22 & 20.6 \\
Distrés psicológico (Escala K-10) & Moderado (16-21) & 33 & 30.8 \\
& Severo (22-29) & 26 & 24.3 \\
& Muy severo (30-50) & 26 & 24.3 \\
Funcionalidad familiar percibida (APGAR - famíliar) & Normo funcional (>7) & 71 & 66.4 \\
& Moderadamente disfuncional (4-6) & 24 & 22.4 \\
& Severamente disfuncional (<3) & 12 & 11.2 \\
\hline Total & & $\mathbf{1 0 7}$ & $\mathbf{1 0 0}$ \\
\hline
\end{tabular}

La tabla 5 indica los valores de la Media y Mediana de la Escala K10. La media está dada por 23.2 puntos y la mediana por 21 puntos, lo que estaría dentro del puntaje dado a distrés moderado. Y la Media y Mediana en el APGAR familiar muestra que la media es 7.3 y la mediana ocho, lo que según el puntaje asignado, cabría dentro de la categoría Normo funcional. 
Tabla 5 - Medias de distrés psicológico y de funcionalidad familiar percibida, por parte de los pacientes atendidos en los Centros de tratamiento del Gran Valparaíso-Chile, 2010

\begin{tabular}{lcccccc}
\hline & & Media & Mediana & Valor mínimo & Valor máximo & Moda \\
\hline K10 Distrés & $\mathrm{n}=107$ & 23.2 & 21 & 10 & 48 & 21 \\
Apgar familiar & $\mathrm{n}=107$ & 7.3 & 8 & - & 10 & 10 \\
\hline
\end{tabular}

\section{Aspectos cualitativos sobre el tratamiento actual percibidos por los pacientes}

\section{Lo que más le gusta}

Con 45 opiniones, los aspectos vinculares con el equipo terapéutico: apoyo en general, comunicación, cariño, disposición, y la profesionalidad de los terapeutas. Con 28 opiniones, las atenciones del centro: charlas, terapias de aclaración de dudas, el aprender a cuidarse, grupos de terapia familiar y grupal. Con 12 opiniones, los aspectos vinculados a funcionamiento del centro: apoyo de terapeutas, terapias de grupo y familiar, y la atención personalizada.

\section{Lo que no le gusta}

Se observa que Nada, tiene 57 respuestas. 20 opiniones refieren que no les gustan aspectos vinculados al funcionamiento del centro, como: tener que adaptarse al centro, mucho tiempo sin actividades, pocas actividades recreativas, corto tiempo de terapia, restricciones para entrar alimentos, ver televisión y usar teléfono, mucha restricción del tiempo para inserción laboral, y visitas restringidas.

\section{Lo que cambiaría}

Nada, tiene 61 respuestas. aspectos vinculados al centro: atención del centro y actividades generales, muestra 15 opiniones: aumentar recursos económicos, horarios de terapias, tiempos más largos de tratamiento, terapias más intensivas, rutinas más dinámicas, seguimientos más intensivos, preocuparse de la reinserción laboral. En atenciones del centro y actividades específicas, con 14 opiniones: terapias al aire libre, deportivas, mejor distribución de las actividades, incorporar otros talleres: biodanza, yoga, más capacitación laboral y Entrega de más herramientas para las recaídas.

\section{DISCUSIÓN}

El General Surgeon Report sobre salud mental de Estados Unidos de 2006, reportó que del 31 a $65 \%$ de los individuos que habían abusado de sustancias alguna vez en la vida, tenían también una historia al menos un desorden mental. ${ }^{7}$ En esta investigación, el $48.6 \%$ de los 107 usuarios investigados reportan distrés psicológico (valores del nivel severo más el nivel muy severo de distrés - psicológico). Existe una serie de factores dentro de las características individuales, propias de la droga y medio ambientales, que interactúan en un paciente con comorbilidad.

En las características individuales, el rango de edad más frecuente de la población en estudio, fue entre 24 y 39 años con un $62.9 \%$, en este rango de edad, el individuo se encuentra en la etapa productiva y de formación de su familia, por lo que el abuso drogas afectará la estructura y el funcionamiento familiar, y será un elemento provocador de distrés psicológico. ${ }^{23}$ Otras características sociodemográficas y económicas de la persona como nivel educativo, empleo, estado marital, e ingresos económicos, aportan otros componentes que podrían influir en la comorbilidad. Puede destacarse como relevante el $45.8 \%$ de solteros, ya que se ha visto que este estado marital es factor de riesgo para la aparición de cuadros que afectan la salud mental, como por ejemplo la depresión. La mayoría de los usuarios, un $51.4 \%$, manifiesta tener un trabajo, lo que podría ser un factor protector, pero a su vez, un $42.1 \%$, refiere no tener ingresos económicos, lo que contrasta con la primera afirmación. La gran mayoría sabe leer y escribir, y 98.1\% ( $n=105)$ tienen buen nivel educacional.

En relación a la percepción de la funcionalidad familiar, contrariamente a lo esperado, en la categoría Normo funcional aparecen la mayoría de los pacientes, es decir $66.4 \%$; luego $22.4 \%$ pacientes perciben a su familia como moderadamente funcional y solo $11.2 \%$ pacientes como Severamente funcional. Esto podría deberse a que el concepto 
de familia funcional como tal, está distorsionado en los personas que abusan de las drogas. ${ }^{24-25} \mathrm{No}$ obstante, todos estos factores interactúan en un contexto donde también confluyen, antecedentes de problemas judiciales relacionados con el consumo de drogas: $48.5 \%$ de los usuarios reportan haber tenido problemas al respecto.

La edad de inicio para el consumo de drogas, por las que principalmente están en tratamiento en los Centros, fue en mayor medida entre 16 y 18 años, siendo la menor edad siete años y la mayor de 41 años, con una media de 21 años. Edad de inicio de consumo de alcohol, fue en mayor medida entre 15 y 18 años, siendo la menor edad siete años, y 50 años la mayor edad, con una media de 16 años. A los 15 años refieren haber iniciado el consumo de otras drogas ilícitas, con una media de 18 años. Antes de iniciar tratamiento, las drogas más consumidas fueron alcohol, un $26.6 \%$, cocaína polvo un 18.2 $\%$ mariguana un $17.2 \%$ y pasta de coca un $16.9 \%$, benzodiacepinas un $5.1 \%$ y anfetaminas un $4.4 \%$. Las drogas por las que mayormente concurren a los Centros de tratamiento los pacientes son: pasta de coca (pasta base) $(41.0 \%)$; la cocaína en polvo $(25.7 \%)$; y el alcohol (23.8\%). Esto concuerda con la prevalencia del consumo de drogas en Chile, en el que estas 3 drogas representan los más altos consumos de la población, junto con la mariguana. ${ }^{19}$

En Chile, no aparece como consumo problemático el uso de drogas inyectables como la heroína o alucinógenos como el LSD. El tipo de atención con que cuentan los pacientes, el equipo de profesionales que los integra, y la satisfacción del usuario con la atención, son aspectos fundamentales que permiten determinar si los Centros de Tratamiento por abuso y dependencia de drogas ofrecen cuidados para la comorbilidad. Los datos resultantes muestran, que de $39.3 \%$ pacientes que manifestaron haber tenido ansiedad antes de ingresar al Centro, $44.9 \%$ han sido diagnosticados luego de ingresar, y 79.2\% están siendo tratados (se entiende que han sido diagnosticados 6 casos nuevos); y en depresión de $45.8 \%$ pacientes que dicen haberla tenido antes del ingreso al Centro, $28.0 \%$ han sido diagnosticados luego de entrar al Centro, estando $76.7 \%$ en tratamiento por este cuadro. No se observa la presencia relevante de otras patologías. La mayoría de los usuarios $88.8 \%$ manifestó estar contento con el tratamiento recibido, $1.9 \%$ no está satisfecho, 3,7\% no sabe y 5.6\% no respondió esta pregunta.

El abordaje adecuado del problema de comorbilidad existente en los consumidores de droga, amerita un diagnostico precoz y un acercamiento terapéutico adecuado, con el fin de poder evitar las complicaciones que puedan aparecer en el proceso de tratamiento y rehabilitación de este paciente. ${ }^{26-27} \mathrm{El}$ consumo de sustancias psicoactivas es un problema que involucra factores de tipo social, educativo, cultural y de salud, y su prevención y tratamiento, debe tener como meta la reinserción del paciente en la sociedad. Esto requiere del permanente esfuerzo de profesionales e instituciones que trabajan en el área.

\section{CONCLUSIONES}

Los resultados indican que como diagnósticos de salud mental previos al ingreso a los centros, se observó con más alta puntuación la Depresión $45.8 \%$, seguido por ansiedad $39.3 \%$ y trastorno bipolar con $4.7 \%$. La mayoría de los pacientes llevaba un año de tratamiento por estos cuadros. Además $74.7 \%$ de los pacientes que buscan los Centros de tratamiento por abuso o dependencia de drogas, tiene comorbidad; con depresión, $28.0 \%$, con ansiedad, $44.9 \%$, y con trastorno bipolar, $1.9 \%$, según fueron diagnosticados en los Centros de tratamiento. Están siendo tratados en estos centros: por depresión, $76.7 \%$ pacientes, por ansiedad, $79.2 \%$ y por trastorno bipolar, $100 \%(n=2)$. De los 107 pacientes hubo $88.8 \%$ que refirieron satisfacción por el tratamiento actual y $48.6 \%$ pacientes presentaron distrés psicológico.

El Apgar familiar indicó que la mayoría de los pacientes $66.4 \%$ pertenece a una familia Normo funcional. Los resultados derivados de esta investigación permitirán afinar y mejorar las intervenciones en pacientes que están en tratamiento y rehabilitación en los Centros de tratamiento.

\section{Limitaciones}

Están relacionadas con el diseño del estudio transversal. Se asume la presencia de un sesgo de supervivencia debido a la naturaleza transversal del diseño, ya que la información que se obtiene corresponde a un momento de vida del paciente. Como consecuencia una visión dinámica de los acontecimientos vitales, como la muerte, emigración, e incidencia de otras enfermedades no se pueden obtener. Otra limitación es el uso de un cuestionario auto-administrado, como la principal fuente de recopilación de datos ya que se consideró que este procedimiento garantiza una mayor confidencialidad y eficacia, pero se espera una mayor tasa de datos faltantes que puede ser 
más prominente entre las personas que tienen dificultades para llenar el cuestionario, debido a los bajos niveles educativos o el desconocimiento de las encuestas.

\section{Recomendaciones}

A la CICAD, debería continuar promoviendo nuevas investigaciones en el ámbito de los Centros de tratamiento y de rehabilitación de usuarios de drogas, donde se incluyan otros países, para poder tener una visión mayor de lo que sucede en América ante el problema de la dependencia y abuso de drogas, su tratamiento y como es abordado.

A las Comisiones Nacionales Antidrogas de los países latinoamericanos, deberían aunar esfuerzos para profesionalizar la atención de los usuarios con abuso de drogas, dar recursos y dictar políticas sobre el tema, apoyando así a los programas de tratamiento y rehabilitación.

\section{AGRADECIMIENTOS}

Esta investigación se realizó gracias al apoyo, asesoría y patrocinio del Gobierno de Canadá, de la Organización de los Estados Americanos (OEA), de la Comisión Interamericana para el Control del Abuso de Drogas (CICAD), y del Centro de Adiciones y Salud Mental (CAMH) Canadá. Asimismo, se agradece la colaboración del personal de los Centros de tratamiento, a los estudiantes que contribuyeron de forma directa o indirecta en el estudio, y especialmente a los pacientes que participaron.

\section{REFERENCIAS}

1. Brady K, Randall C. Gender differences in substance use disorders. Psychiatr Clin North Am. 1999 Jun; 22(2):241-52.

2. Drake R, Mueser KT, Brunette M, McHugo GJ. A review of treatments for people with severe mental illnesses and co-occurring substance use disorders. Psychiatr Rehabil J. 2004 Spring; 27(4):360-74.

3. Conway KP, Compton W, Stinson FS, Grant BF. Lifetime comorbidity of DSM-IV mood and anxiety disorders and specific drug use disorders: results from the National Epidemiologic Survey on Alcohol and Related Conditions. J Clin Psychiatry. 2006 Feb;67(2):247-57.

4. Clark RE. Family support and substance use outcomes for persons with mental illness and substance use disorders. Schizophr Bull. 2001; 27(1):93-101.

5. Drake R, McLaughlin P, Pepper B, Minkoff K. Dual diagnosis of major mental illness and substance disorder: an overview. New Dir Ment Health Serv. 1991 Sum; (50):3-12.

6. Australian Bureau of Statics. Use of the Kessler psychological distréss scale in ABS health and wellbeing survey, psychological distréss in the Western Australian population 1997 and 2001 [online]. Australia: ABS; 2001 [acessed 2009 Jun 15]. Disponible en: www.abs.gov.au/ausstats/abs@. nsf/.../4817.0.55.001

7. Colin C. Substance abuse issues and public policy in Canada. Canada's federal drug Strategy. 2006 [acesso 2009 Jun 25]. Disponible en: www.parl.gc.ca/ information/library/PRBpubs//prb0615-e.html

8. Evans K, Sullivan JM. Dual diagnosis. counseling the mentally III substance abuser. New York (US): The Guilford Press; 2001.

9. Dalex DC, Moss HB. Dual disorders. counseling clients with chemical dependency and mental illness. $3^{\mathrm{a}}$ ed. Minnesota (US): Hazelden Foundation Center; 2002.

10. Brady S, Hiam CM, Saemann R, Humbert L, Fleming M, Dawkins-Brickhouse K. Dual diagnosis: a treatment model for substance abuse and major mental illness. Community Ment Health J. 1996 Dec; 32(6):573-8.

11. Skinner WJW, editor. Treating concurrent disorders a guide for counselors. Toronto (CA): Centre for addiction and Mental Health; 2005.

12. Kessler RC, Nelson CB, McGonagle KA, Edlund MJ, Frank RG, Leaf PJ. The epidemiology of co-occurring addictive and mental disorders: implications for prevention and service utilization. Am J Orthopsychiatry. 1996 Jan;66(1):17-31.

13. Kranzler HR, Mason B, Modesto-Lowe V. Prevalence, diagnosis, and treatment of comorbid mood disorders and alcoholism. In: Kranzler HR, Rounsaville B editors. Dual diagnosis and treatment. New York (US): Marcel Dekker, Inc. 1998. p. 107-36.

14. Dixon L, McNary S, Lehman A. Substance abuse and family relationships of persons with severe mental illness. Am J Psychiatry. 1995 Mar; 152(3):456-8.

15. Drake R, Mueser K, Clark R, Wallach M. The course, treatment, and outcome of substance disorder in persons with severe mental illness. Am J Orthopsychiatry. 1996 Jan; 66(1):42-51.

16. Carey K. Substance use reduction in the context of outpatient psychiatric treatment: a collaborative, motivational, harm reduction approach. Community Ment Health J. 1996 Jun; 32(3):291-306; discussion 307-10.

17. Grant BF. The influence of comorbid major depression and substance use disorders on alcohol and drug treatment: results of a national survey. In: National Institute on Drug Abuse (NIDA). Treatment of drug-dependent individuals with comorbid mental disorders. Rockville (US): NIH; 1997. 
18. Organización de los Estados Americanos, Comisión Interamericana para el Control del Abuso de Drogas. Chile, Grupo de expertos gubernamentales: Evaluación del Progreso de Control de Drogas, 2003-2004. Mecanismo de Evaluación Multilateral. Washington D.C. (US): OEA, CICAD; 2006.

19. Gobierno del Chile, Ministerio del Interior. Octavo estudio nacional de drogas en población general de Chile, 2008 [en línea]. 2009. Disponíble en: www. conace.cl/.../OctavoEstudioDrogas2008.pdf

20. Minoletti A, López C. Las enfermedades mentales en Chile. Santiago (CL): Ministerio de Salud, Unidad de Salud Mental; 1999.

21. Smilkstein G. The family APGAR: a proposal for family function test and its use by physicians. J Fam Prac.1978 Jun; 6(6):1231-9.

22. Smilkstein G, Ashworth C, Montano D. Validity and reliability of the family APGAR as a test of family function. J Fam Prac. 1982 Aug; 15(2):303-11.

23. Kessler RC, Andrews G, Colpe LJ, Hiripi E, Mroczek DK, Normand SLT, et al. Short screening scales to monitor population prevalances and trends in non- specific psychological distréss. Psychol Medicine. 2002 Aug; 32(6):959-76.

24. Basso L. Grado de satisfacción de la funcionalidad familia en adolescentes entre 12 y 18 años consumidores de drogas [tesis]. Valparaíso (CL): Universidad de Valparaíso: Escuela de Enfermería. Facultad de Medicin, 2008.

25. Rodríguez André y otros. ¿Tienen apoyo social y familiar los drogodependientes que participan en el programa «libre de drogas» en prisión? Medicina de Familia [online]. $2004 \mathrm{Feb}$ [acesso 2009 Nov 15]; 5(1). Disponível em: http://www.samfyc.es/Revista/ PDF/v5n1/04.pdf

26. Pratt L, Dey A, Cohen A. Characteristics of adults with serious psychological distréss as measured by the K6 Scale: United States, 2001-04. Adv Data. 2007 Mar 30; (382):1-18.

27. Rush BR, Castel S, Brands B, Toneatto T. Validation and comparison of screening tools for mental disorders in substance abusers. In: Abstract Book of the Annual Meeting of the College on Problems of Drug Dependence, 2008, june $14^{\text {th }}-19^{\text {th }}$; San Juan, Puerto Rico: CPDD; 2008. 